\title{
Decomposing the causes of inequalities in health care use: a micro-simulations approach
}

\author{
Hélène HUBER*
}

August 6, 2008

\begin{abstract}
We propose an innovative method for the decomposition of factors associated with inequalities in the use of health care. We analyze individual data and make use of microsimulations to evaluate the effect of heterogeneity of individual behaviors on inequality in access to care. Our study employs methods that, unlike earlier work, permits evaluation of heterogeneity of individual behaviors. We provide an application of this method by decomposing inequality of health care use in France in 1998. We show that half of the inequity in access to care is due to the heterogeneity of behaviors relative to the rank of individuals in the income distribution. This approach reconciles Oaxacalike decompositions of inequality, focused on outcome gaps, with analyses involving decompositions of inequality by factors, focused on inequity indices.
\end{abstract}

Keywords: Health care consumption - Econometrics - Microsimulations - Inequalities JEL codes: C15 - C34 - D63 - I11 - I18

I would like to thank Brigitte Dormont, Pierre-Yves Geoffard, Myriam Khlat, Marc Perronnin, Lise Rochaix, Anne-Laure Samson and Sandy Tubeuf for their help and useful comments throughout the development of this paper. I also thank the editor, two anonymous reviewers as well as Tim-Allen Bruckner whose remarks helped improve the paper. I am grateful to the IRDES team for their help in accessing their data. All errors are my responsibility.

* Paris School of Economics, Université Paris 1 Panthéon-Sorbonne, Centre d'Economie de la Sorbonne, TEAM, 106-112 bld de l'Hôpital, 75647 Paris Cedex 13, France - helene.huber@univ-paris1.fr 


\section{Introduction}

The issue of inequalities in health and health care use is regularly part of the public agenda. In European countries, the goal of public policies is not only to globally improve the health status of the population, but also to ensure equality in access to care. In most of these countries, the main concept of the health care system is egalitarian: health care is allocated according to individual need ${ }^{1}$, and is meant to be dissociated from the ability to pay for health care. For Wagstaff and VanDoorslaer (2000), the literature concerning equity in access to care is strongly related to the political agenda, which could explain the great amount of European literature on this theme. For these authors, equity of the health care system is conceived as a fundamental objective even more important than its efficiency. Equity in health care use usually refers to horizontal equity, which will be the focus of this paper. Horizontal equity is defined by a situation where on average, people in the same need of treatment receive a similar treatment, irrespective of their other characteristics (notably income $)^{2}$.

While the French health care system is structured as egalitarian, covering $99 \%$ of the population (Régime Général) and with mostly regulated fees, there still exist inequalities in health and health care use (VanDoorslaer and Masseria 2004). In addition, even for those covered, there still exist financial barriers to care, notably the absence of complementary insurance, or the presence of excess fees (in excess of the regulated fee amount) that some medical doctors are allowed to demand.

For example, a great number of studies (VanDoorslaer and Masseria 2004, VanDoorslaer, Wagstaff, Burg, Christiansen, Graeve, Duchesne, Gerdtham, Gerfin, Geurts, Gross, Hakkinen, John, Klavus, Leu, Nolan, O’Donnell, Propper, Puffer, Schellhorn, Sundberg, and Winkelhake 2000, VanDoorslaer, Koolman, and Jones 2004) show that while health care needs are concentrated among the poor, health care use (mostly specialists visits) is concentrated among the rich. This particular feature of specialist visits can be found in many European countries. Income inequality is often depicted as a major cause for this mismatch between health care need and use of health care.

However, if all the financial barriers to care were eliminated, the health care distribution would not necessarily become totally equitable. Many factors can render the distribution of health care inequitable. For example, agents' preferences may differ ; some can prioritize health more than others. Individuals can also disagree on the benefit provided by health care use, or on the evaluation of this benefit. Heterogeneity of preferences on the one hand, and the trade-off between costs and benefit on the other, could explain the apparent inequity in health care use, for a given health status. The observed inequity would then result from rational choices.

\footnotetext{
${ }^{1}$ A discussion of the notion of need for care can be found in Wagstaff and VanDoorslaer (2000).

${ }^{2}$ Vertical equity is defined by a situation where people in different need for care receive appropriately dissimilar treatments (Wagstaff and VanDoorslaer 2000).
} 
Wagstaff and VanDoorslaer (2000) contend that an equitable health care distribution should exactly reflect health care need in the population ${ }^{3}$. Consequently, any deviation from this needexpected distribution is inequitable. Inequalities in health care use can thus be decomposed into a "justifiable" part, due to the distribution of need, and an "unjustifiable" part, due to all other factors. A measure of inequality in health care use (often its concentration index with respect to individual income) is usually decomposed into the sum of the different factors which have led to this inequality, labeled justifiable (e.g. differences in health care need) or unjustifiable (e.g. differences in income). However, these decompositions generally account for differences in individual characteristics and endowments (income level, health status or education), but they do not account for differences in individual behaviors.

In this article we propose an innovative method for the decomposition of inequalities in health care use. The main contribution of this work is to extend the standard methodology by proposing a decomposition that allows for the evaluation of the impact of individual preferences on inequalities in access to care. We take into account the impact of the heterogeneity of behaviors with respect to the individuals' social rank, which cannot be taken into consideration in the standard methodology.

We first describe the limitations of the standard methodology. Second, we describe our methodology and how it addresses these limitations. Third, we present an application using French data from 1998 (the Appariement developed by $\operatorname{IRDES}^{4}$ ), that decomposes factors associated with inequality in health care use.

\section{The decomposition of inequalities in health care use: the standard method and its limitations}

\subsection{The concentration index and its decomposition}

An index measuring inequality in health care use with respect to income must be able to describe observed inequality, and identify its "justifiable" and "unjustifiable" parts. The inequality index we use here is the concentration index of health care use, relative to income. This index is widely used and has convenient properties (Wagstaff and VanDoorslaer 2000). In particular, it can be easily decomposed in a linear way (see section 2.1). It is used in particular by ECUITY ${ }^{5}$, a group devoted

\footnotetext{
${ }^{3}$ From this point of view, health inequalities are described as given, while one should keep in mind that the distribution of health is likely to be influenced by health care use.

${ }^{4}$ Institute for research and information in health economics, 10 rue Vauvenargues, 75018 Paris, France - www. irdes.fr

${ }^{5}$ European project whose goal is to study inequality in health, health care use and health care financing: http: //www2.eur.nl/bmg/ecuity/. Research teams come from the following countries: Belgium, Denmark, Finland, France, Germany, Greece, Ireland, Italy, the Netherlands, Spain, Sweden, U-K, Norway, Switzerland, USA.
} 
to international comparisons in health and health care use inequalities.

The concentration index of a variable $y$ (for example, the number of physician visits) relative to income $I$ can be defined using the concentration curve of $y$ with respect to $I$. This concentration curve links the cumulative proportion of individuals, ranked by income, to the corresponding proportion of $y$ used by them, with respect to the total amount of $y$. Within this framework, a measure of inequality in health care use is given by the concentration index, i.e. twice the area between the concentration curve and the line of equality. Let there be a population of $n$ individuals $i(i \in[1 . . n])$, each having a health consumption $y_{i}$ and whose fractional $\mathrm{rank}^{6}$ in the income distribution is $R_{i}$. Let $\bar{y}$ be the mean health care use. The concentration index $C$ of health care use in this population can be defined as $C=\frac{2}{n \bar{y}} \sum_{i=1}^{n} y_{i} R_{i}-1$ (Wagstaff, VanDoorslaer, and Watanabe 2003).

We should emphasize here that inequality can be measured with tools other than the concentration index. Since we do not use the standard decomposition method in our new methodology when disentangling the various factors leading to inequality, the exclusive use of the concentration index is no longer necessary. We nevertheless use it for comparability purposes.

Assume here that health care use can be explained by factors $\left(x_{k}\right), k \in[1 . . K]$ within a standard linear model (Wagstaff, VanDoorslaer, and Watanabe 2003), which is a rather strong assumption:

$$
\forall i \in[1 . . n], y_{i}=\alpha+\sum_{k=1}^{K} \gamma_{k} x_{k, i}+\varepsilon_{i}
$$

This implies the following decomposition (Wagstaff, VanDoorslaer, and Watanabe 2003) ${ }^{7}$ :

$$
C=\sum_{k=1}^{K} \frac{\gamma_{k} \overline{x_{k}}}{\bar{y}} C_{k}+\frac{G C_{\varepsilon}}{\bar{y}}
$$

Where $\overline{x_{k}}$ is the mean of the $x_{k, i}, \bar{y}$ the mean of $y_{i}, C_{k}$ the concentration index of $x_{k}$ with respect to income, and $G C_{\varepsilon}$ the generalized concentration index of $\varepsilon_{i}\left(G C_{\varepsilon}=\frac{2}{n} \sum_{i=1}^{n} \varepsilon_{i} R_{i}\right)$.

\subsection{The Horizontal Inequity Index and its drawbacks}

\subsubsection{Computation of the Horizontal Inequity index}

In expression (2), labeling $M$ the need variables and $Z$ all the other variables, and labeling $\delta$ and $\beta$ their vectors of coefficients yields:

\footnotetext{
${ }^{6}$ Let $r_{i}$ be the rank of individual $i$ in the income distribution. The fractional rank $R_{i}$ is defined as $R_{i}=r_{i} / n$.

${ }^{7}$ Originally, Wagstaff, VanDoorslaer, and Watanabe (2003) had developed this decomposition for health inequality and not health care use inequality, but their method is suitable for the decomposition of inequality of any variable $y$ (see VanDoorslaer and Masseria (2004)).
} 


$$
C=\sum_{k=1}^{K_{1}} \frac{\delta_{k} \overline{m_{k}}}{\mu} C_{k}+\sum_{k=1}^{K_{2}} \frac{\beta_{k} \overline{z_{k}}}{\mu} C_{k}+\frac{G C_{\varepsilon}}{\mu} .
$$

The first element on the right hand side of expression (3) represents justifiable inequality, i.e. inequality in need. The second and third elements jointly represent unjustifiable inequality, arising from factors other than need: their sum equals the Horizontal Inequity Index. Inequality in health care use with respect to income could totally be explained by differing needs: if the distribution of health care use perfectly reflects the distribution of need, the distribution of health care use can be inegalitarian but equitable.

\subsubsection{Limitations}

Linearity The decomposition as expressed in (2) is necessarily linear. However, a great number of explanatory models of health care use are non linear (for example, count data models). In this case, decomposition (2) is used, with parameters $\gamma_{k}$ replaced by parameters $\gamma_{k}^{m}$, where the $\gamma_{k}^{m}$ are the marginal effects of each variable $x_{k}$ evaluated at the sample mean (WorldBank 2005). The decomposition is then an approximation. VanDoorslaer and Masseria (2004) recommend the use of a standard linear model such as (1) for $y$, even when the estimated model does not suit the variable. Although quite convenient, this solution is conceptually unsatisfactory.

The interpretation of the $C_{k}$ Expressions (2) and (3) involve the concentration indices of every explanatory factor, which implies that these indices make sense. Among other variables, indicators of health status are present in the explanatory model of health care use. Computing the concentration index on a continuous health variable means considering it as an "ill-health stock", that could be aggregated into a total stock of ill-health at the population level. This is obviously not the case for a categorical variable. To avoid such a misuse of the variable, one can compute a concentration index for every category of the health indicator considered (each category being coded with a dummy variable: 0/1). This option is chosen by the ECUITY group. The main drawback of such a method is that describing the distribution of an indicator of ill-health according to income becomes difficult, as there are as many concentration indices to analyze as there are categories within this indicator. The new method we propose here avoids the computation and interpretation problems of such indicators. In addition to the issue concerning health indicators, income is often introduced as its logarithm in the explanatory models; the standard decomposition leads to the computation and interpretation of the concentration index of the $\log$ of income with respect to income itself, which makes interpretation difficult.

Accounting for preferences Disentangling inequality into its justifiable and unjustifiable parts using expression (3) assumes that an equitable distribution of health care use is the one that we would observe if every individual in the population were treated like the average individual, ac- 
cording to his/her health status. Indeed, to implement decompositions (2) then (3), the estimation of model (1) is made on the whole sample. For any indicator of health care need $m_{k}$, the estimated

coefficient $\hat{\delta_{k}}$ represents what amount of health care society as a whole allocates on average for that need. Any deviation from this average relationship is taken into account by variables $Z$ and their coefficients $\beta$. In the ECUITY group studies, this average behavior is taken as a norm. One cannot distinguish different $\delta_{k}$ by income level, and presumably different preferences according to income level. Indeed, the social epidemiology literature has shown that individuals' social rank has an impact on health behaviors (Wilkinson 1992). Decomposition (3) only takes into account the distribution of explanatory variables with respect to income, and does not take into account the potential heterogeneity of coefficients with respect to income.

The limitation to a single explanatory equation of health care consumption Explanatory models of health care consumption can be highly non-linear. The most relevant - and common - model specifications are two-part models, explaining the decision to consume (probit or logit) and the level of consumption conditional on the participation to the health care system (GLM truncated at zero). Another way of modeling health care consumption is the use of single equation zero-inflated models.

Whatever explanatory model is chosen, the decomposition of inequality into several factors (expression (2) or its approximation in the case of a non-linear model) can only be computed for one single equation of a model and is a linear approximation. In the works of ECUITY, we thus get separately:

- a decomposition of the probability of use (logit or probit model)

- a decomposition of the conditional consumption (GLM or $O L S)$

- a decomposition of unconditional consumption (zero-inflated model).

Consistency between the different steps of the reasoning is not guaranteed: two-part models and zero-inflated models are conceptually different and do not model the same processes. Consequently, this method does not ensure that the fraction of inequity due to a certain factor can be relevantly partitioned into participation and conditional consumption behaviors.

\section{A new method using microsimulations}

The new decomposition method proposed here overcomes the difficulties of the standard approach (see section 2). To allow for comparison of our results to those of the ECUiTy group, we chose the widely used concentration index as the measurement of inequity. 
We run convenient estimation methods of health care use first on the whole sample, then on the different income quantiles of the sample. Next, we compute counterfactual distributions of health care use, assuming various hypotheses detailed below, notably homogeneity then heterogeneity of behaviors. This computation and comparison of counterfactual health care use distributions is inspired from Dormont, Grignon, and Huber (2006). For all these simulated distributions, we compute the concentration index of health care use with respect to income; we are thus able to identify the impact of each hypothesis on the value of the concentration index. This innovative method provides several advantages: it avoids the linear approximation that is imposed by the standard decomposition approach even when the explanatory model is non-linear, it avoids the limitation to one single equation and it permits the identification of the impact of heterogeneity of behaviors on the observed inequality in health care use.

While the standard decomposition expresses the health care use concentration index as a function of the concentration indices of all the explanatory factors of the model (see expression (2)), the method we propose here does not require explanatory factors of health care use whose concentration index makes sense.

We simulate health care use distributions from which we compute the concentration index instead of decomposing the concentration index of an observed distribution. Contrary to the standard method, this process allows us to disentangle the effects of participation and conditional consumption within one single explanatory model of total health care consumption.

The most innovative feature of our method is the ability to estimate a model of health care use by income quantile. We propose a horizontal inequity index that depends not only on the distribution of variables $Z$ according to income (already provided by the standard method) but also on the heterogeneity of parameters $\beta$ and $\delta$ with respect to income. We believe that this method provides a way to identify individual preferences, which is not possible with the standard decomposition described in section 2.

\subsection{Modeling health care use}

Let $y_{i}$ be the variable representing individual $i$ 's health care use within a population of $N$ individuals. For example, $y_{i}$ can take the format of a number of visits, or expenditures. We choose here to use a modified two-part model: Logit for the participation equation and a GLM for the consumption equation.

As is common in the literature, explanatory variables are categorized as need ( $M_{1}$ and $M_{2}$ for participation and consumption equation respectively) and non-need variables $\left(Z_{1}\right.$ and $Z_{2}$ respec- 
tively). The explanatory model is the following:

(1) $E\left(P_{i}\right)=G\left(Z_{1} b+M_{1} d\right)$

(2) $E\left(y_{i} / P_{i}=1\right)=F\left(Z_{2} \beta+M_{2} \delta\right)$

A prediction of the model for every individual $i$ is as follows:

$$
\widehat{y_{i}}=\underbrace{G\left(Z_{1, i} \widehat{b}+M_{1, i} \widehat{d}\right)}_{\text {participation }} * \underbrace{F\left(Z_{2, i} \widehat{\beta}+M_{2, i} \widehat{\delta}\right)}_{\text {conditional consumption }}
$$

Let $C$ be the concentration index of health care use $y$ in the observed population. The objective here is to decompose the value of $C$ into the unique contributions of each group of variables, using simulated distributions of health care use.

\subsection{Parameter estimation}

The model is estimated on the whole sample, yielding parameters $\widehat{b}, \widehat{d}, \widehat{\beta}$ and $\widehat{\delta}$. The sample is then split into $Q$ income quantiles. The model is estimated on each quantile, and the following parameters are computed: $\left(\widehat{b_{q}}\right),\left(\widehat{d_{q}}\right),\left(\widehat{\beta_{q}}\right)$ and $\left(\widehat{\delta_{q}}\right), q \in[1 \ldots Q]$. Parameters $\widehat{\delta_{q}}$ can differ, which would indicate the presence of different practices for a given morbidity, according to the income quantile to which the individual belongs. Parameters $\widehat{\beta_{q}}$ can differ as well, which would indicate a heterogenous effect of socio-economic variables on health care consumption, with respect to income level. This holds as well for parameters $\widehat{d_{q}}$ and $\widehat{b_{q}}$. This estimation of coefficients specific to distinct subgroups of population is directly inspired from Oaxaca (1973). Income level is controlled for at every step.

For each estimated equation, the parameters represent individual behaviors. Parameters $d$ and $\delta$ (as well as their equivalents $d_{q}$ and $\delta_{q}$ for each income quantile $q$ ) represent the observed practices for a given level of morbidity, all other things equal. In the remainder of the article, we will call these parameters practices: the value of a parameter estimate represents the response in terms of health care to a given health status. These practices are individual behaviors, and can originate from the demand or supply of health goods. Parameters $d$ correspond to practices linked to participation, which can be related to demand. Parameters $\delta$ correspond to practices linked to conditional consumption, mainly related to supply. 


\subsection{The simulation of distributions and the computation of their con- centration indices}

Once parameters are estimated, we simulate health care use distributions under various hypotheses. We label $\overline{Z_{1}}, \overline{M_{1}}, \overline{Z_{2}}$ and $\overline{M_{2}}$ the sample mean of each corresponding variable. To understand the role of each factor in total observed inequality in health care use, we assume a perfectly egalitarian distribution of health care use. Then, we change one by one each explanatory factor of health care use in order to identify its contribution to the degree of inequality that is observed for variable $y_{i}$. The different steps of simulations are described hereafter.

The baseline of our microsimulations is a distribution of health care use that is perfectly egalitarian. It corresponds to a situation where all the individuals would share the same characteristics $\left(\forall i, \forall j \in\{1,2\}, Z_{j, i}=\overline{Z_{j}}\right.$ and $\left.M_{j, i}=\overline{M_{j}}\right)$ and where the impact of these characteristics would be the same, regardless of the income quantile of the individual $\left(\forall q, b_{q}=b\right.$ and $d_{q}=d$ et $\forall q, \beta_{q}=\beta$ and $\delta_{q}=\delta$ ). This distribution of health care use can be expressed as follows:

$$
\forall i, \widehat{y_{i}^{0}}=G\left(\overline{Z_{1}} \widehat{b}+\overline{M_{1}} \widehat{d}\right) * F\left(\overline{Z_{2}} \widehat{\beta}+\overline{M_{2}} \widehat{\delta}\right)=\text { Constant }
$$

As the simulated health care use is by definition the same for everyone, its concentration index is necessarily equal to $0: C I_{0}=0$.

The next step of the simulation takes into account the heterogeneity of morbidity among individuals, while keeping behaviors homogenous:

$$
\forall i, \widehat{y_{i}^{1}}=G\left(\overline{Z_{1}} \widehat{b}+M_{1, i} \widehat{d}\right) * F\left(\overline{Z_{2}} \widehat{\beta}+M_{2, i} \widehat{\delta}\right)
$$

Where $\overline{M_{1}}$ is replaced by $M_{1, i}$ and $\overline{M_{2}}$ by $M_{2, i}$ in expression (7). The concentration index $C I_{1}$ computed on distribution $\widehat{y^{1}}$ gives the degree of inequality due to inequality in morbidity. It is close conceptually to the Need index, mentioned in section 2.2 , because it represents the index of inequality that is due to diffences in need with respect to income.

Following this reasoning, every element of the right hand side of equation (7) (variables and parameters alike) is successively replaced in order to eventually get to the final observed distribution. The following table summarizes the whole process. The name of the concentration index computed on each counterfactual distribution is labeled next to each equation. 


$$
\begin{array}{rcl}
\text { Baseline } & \widehat{y_{i}^{0}}=G\left(\overline{Z_{1}} \widehat{b}+\overline{M_{1}} \widehat{d}\right) * F\left(\overline{Z_{2}} \widehat{\beta}+\overline{M_{2}} \widehat{\delta}\right) & C I_{0} \\
\text { Morbidity } & \widehat{y_{i}^{1}}=G\left(\overline{Z_{1}} \widehat{b}+M_{1, i} \widehat{d}\right) * F\left(\overline{Z_{2}} \widehat{\beta}+M_{2, i} \widehat{\delta}\right) & C I_{1} \\
\text { Practices (part.) } & \widehat{y_{i, q}^{2}}=G\left(\overline{Z_{1} \widehat{b}}+M_{1, i} \widehat{d_{q}}\right) * F\left(\overline{Z_{2}} \widehat{\beta}+M_{2, i} \widehat{\delta}\right) & C I_{2} \\
\text { Practices (cond. cons.) } & \widehat{y_{i, q}^{2 b i s}}=G\left(\overline{Z_{1} \widehat{b}}+M_{1, i} \widehat{d_{q}}\right) * F\left(\overline{Z_{2}} \widehat{\beta}+M_{2, i} \widehat{\delta_{q}}\right) & C I_{2 b i s} \\
\text { SES indicators } & \widehat{y_{i, q}^{3}}=G\left(Z_{1, i} \widehat{b}+M_{1, i} \widehat{d_{q}}\right) * F\left(Z_{2, i} \widehat{\beta}+M_{2, i} \widehat{\delta_{q}}\right) & C I_{3} \\
\text { Impact of SES } & \widehat{y_{i, q}^{4}}=G\left(Z_{1, i} \widehat{b_{q}}+M_{1, i} \widehat{d_{q}}\right) * F\left(Z_{2, i} \widehat{\beta_{q}}+M_{2, i} \widehat{\delta_{q}}\right) & C I_{4}
\end{array}
$$

Notice that accounting for the heterogeneity of practices corresponding to participation is made in equation (9) while the one corresponding to the conditional consumption is made in equation (10).

By calling $C$ the observed concentration index computed on observed health care use, it can be easily seen that $C$ is equal to the sum of the following 6 elements:

- $\Delta_{1}=C I_{1}-C I_{0}\left(=C I_{1}-0=C I_{1}\right)$ is the contribution of the inequalities in morbidity

- $\Delta_{2}=C I_{2}-C I_{1}$ is the contribution of the heterogeneity of practices corresponding to participation

- $\Delta_{2 b i s}=C I_{2 b i s}-C I_{2}$ is the contribution of the heterogeneity of practices corresponding to the conditional consumption

- $\Delta_{3}=C I_{3}-C_{2 b i s}$ is the contribution of socio-economic inequalities

- $\Delta_{4}=C I_{4}-C I_{3}$ is the contribution of the heterogeneity of behaviors linked to the socioeconomic variables

- $\Delta_{5}=C-C I_{4}$ is the contribution of unobserved heterogeneity

And $C=\Delta_{1}+\Delta_{2}+\Delta_{2 b i s}+\Delta_{3}+\Delta_{4}+\Delta_{5}$.

\subsection{Interpretation}

The component of $C$ due to the heterogeneity of needs is equal to $\Delta_{1}$, that we will call Need in what follows, mainly in order to compare our results with the existing literature (see equation (3)):

$$
\text { Need }=\Delta_{1}
$$

The component of $C$ due to other factors than need, labeled $H I$, is equal to $\Delta_{2}+\Delta_{2 b i s}+\Delta_{3}+$ $\Delta_{4}+\Delta_{5}:$ 


$$
H I=\Delta_{2}+\Delta_{2 b i s}+\Delta_{3}+\Delta_{4}+\Delta_{5} .
$$

We should emphasize that the effect of unobserved heterogeneity is contained within $H I$ (see VanDoorslaer and Masseria (2004)).

This decomposition quantifies the global effect of morbidity on the degree of inequality observed (the Need indicator), and does not have the computation and interpretation issues of the $C_{k}$ that are found in expression (2). Moreover, whereas the heterogeneity of practices is not modeled in the standard approach, it is explicitly modeled here with the estimated parameters $\widehat{d_{q}}$ and $\widehat{\delta_{q}}, q \in[1 . . Q]$. As was mentioned earlier, the groups of variables we chose reflect the common distinction between need and non-need variables. However, our method is able to compute the contribution to inequality of any group of variables or even any isolated variable.

Index $H I$ as defined by expression (14) can be decomposed into two components : one due to the distribution of the variables in the sample and one due to the heterogeneity of the coefficients corresponding to these variables. The component of inequality due to the variables is thus defined by the following expression:

$$
\text { Variables }=\Delta_{3}+\Delta_{6}
$$

While the component of $H I$ due to the heterogeneity of behaviors can be written as:

$$
\text { Behaviors }=\Delta_{2}+\Delta_{2 b i s}+\Delta_{4}
$$

The degree of inequality due to the heterogeneity of behaviors with respect to income can be decomposed into three effects:

- $\Delta_{2}$ : practices corresponding to participation, that can be linked to the demand side

- $\Delta_{2 b i s}$ : practices corresponding to the conditional consumption, that can be linked mainly to the supply side

- $\Delta_{4}$ : parameters corresponding to non-need variables (socio-economic variables), that can be linked to the demand side

This decomposition enables us to distinguish the degree of inequality that is due to the supply and demand of health care through the analysis of $\Delta_{2}$ and $\Delta_{2 b i s}$ and to identify precisely the agents' preferences. 


\section{The analysis of inequality in health care use in France: an application}

We use the Appariement, a survey conducted in France every other year by IRDES. We use year 1998 and restricted the analysis to people age 15 and above. The data provide detailed health care use of every individual, as well as personal characteristics such as age, household size and household income. A great number of morbidity indicators are available, such as synthetic indicators (e.g. level of disability) as well as detailed indicators corresponding to diseases (e.g. presence of diabetes). The data comprise 4,336 individuals. We focus on the number of visits, divided into the number of general practitioner (GP) visits and the number of specialist visits ${ }^{8}$. Average number of visits is 4,8 for GPs and 3,5 for specialists. Detailed results of analyses are available upon request. We decompose inequality in health care use using the new method we outlined in section 3 , and compare our results to those yielded from the standard decomposition method.

\subsection{Decomposition process}

\subsubsection{The choice of a model for health care use}

We use a two-part model, which explains participation in the health care system and conditional consumption for users with two independent equations. The dependent variable is the number of visits (GPs, specialists and dentists, total). The explanatory variables of health care use are selected according to their degree of significance in a regression explaining the total number of visits. We chose a common set of variables to explain GP and specialist visits, in order to easily compare results. Since individuals are observed for a one year period, the synthetic indicators of morbidity as well as the presence of complementary insurance are likely to be endogenous in the estimated equations: only exogenous variables are kept in the analysis. This selection of variables is performed on the whole sample of individuals age 15 and above. The detailed list of selected variables is available upon request.

We model the probability of participation to consultations using a Logit model. To model the conditional number of visits we choose a GLM (Generalized Linear Model) using a log link and a Zero-Truncated Negative Binomial as the distribution. Indeed, the data show overdispersion in that the variance of the dependent variable is greater than its expected value (see VanDoorslaer, Koolman, and Jones (2004) or Grogger and Carson (1991)). We categorized the sample into income quintiles $q \in[1 . . Q]$, with $Q=5$.

\footnotetext{
${ }^{8}$ The numbers of visits were computed by the author using IRDES data.
} 


\subsection{The analysis of inequalities in health care use in France, year 1998}

\subsubsection{Results using the new methodology}

The results are presented here as tables synthesizing total inequality, decomposed into various elements. The degree of total inequality, called $C$, is presented as the sum of two elements: the degree of inequality due to the heterogeneity of need ${ }^{9}$ (the Need index) and the degree of inequality due to other factors than need (the inequity index $H I$ ). The inequity index $H I$ is in turn decomposed into the distribution of variables with respect to income, and the heterogeneity of behaviors (represented by the heterogeneity of coefficients with respect to income). This behavioral component comes from the heterogeneity of three types of coefficients, whose different effects are presented in the tables:

- the heterogeneity of practices corresponding to participation (linked to need),

- the heterogeneity of practices corresponding to conditional consumption (linked to need),

- the heterogeneity of other behaviors (linked to the other non-need variables).

The table presented hereafter provides the components of the decomposition of inequality observed on our sample, year 1998. In each row, the value written in bold is equal to the sum of the components written as plain text in that same row.

\begin{tabular}{r|r|r|r} 
& GP's & Specialists & All physicians \\
\hline $\mathbf{C}$ & $\mathbf{- 0 , 0 2 1 9}$ & $\mathbf{0 , 0 8 8 4}$ & $\mathbf{0 , 0 2 5 2}$ \\
Need & $-0,0047$ & $-0,0078$ & $-0,0065$ \\
HI & $-0,0172$ & 0,0962 & 0,0317 \\
\hline HI & $\mathbf{- 0 , 0 1 7 2}$ & $\mathbf{0 , 0 9 6 2}$ & $\mathbf{0 , 0 3 1 7}$ \\
Variables & $-0,0097$ & 0,0450 & 0,0135 \\
Behaviors & $-0,0075$ & 0,0512 & 0,0182 \\
\hline & & & \\
Behaviors & $\mathbf{- 0 , 0 0 7 5}$ & $\mathbf{0 , 0 5 1 2}$ & $\mathbf{0 , 0 1 8 2}$ \\
Participation & 0,0004 & 0,0381 & 0,0257 \\
Other & 0,0281 & 0,0481 & 0,0047 \\
Cond. consumption & $-0,0361$ & $-0,0351$ & $-0,0122$
\end{tabular}

Table 1: Decomposition of the inequality observed in 1998 in France

Let us recall that "participation" and "other" behaviors relate to the demand side, while "conditional consumption" behaviors relate to the supply side.

The contribution of each factor was computed using the concentration indices of the various simulated distributions of health care use. An analysis of robustness with respect to the order of introduction of the various effects was performed ; results remained essentially unchanged.

\footnotetext{
${ }^{9}$ In other words, due to morbidity and age.
} 


\subsubsection{Results obtained with the standard methodology}

We implemented the standard methodology on our data, using the number of GP visits as the outcome variable. As is common practice in the ECUITY research papers to take into account the mass in zero, we run Zero-Inflated regressions and computed the marginal effects of each variable in order to get the decomposition given by expression (2) using marginal effects (see section 2.2.2). The total index of inequality $C$ is decomposed into the sum of one part due to need (Need) and another part due to all the non-need factors $(H I)$. Results are given in the following table :

\begin{tabular}{r|r|r} 
& Value & Percentage \\
\hline $\mathrm{C}$ & $-0,022$ & \\
\hline Need & $-0,018$ & 0,82 \\
HI & $-0,004$ & 0,18
\end{tabular}

Table 2: Decomposition of the inequality in GP visits, year 1998, using the ECUITY methodology

The order of magnitude of the indices of total inequality $C$ is similar to other studies (see for example VanDoorslaer and Masseria (2004), on Appariement data of year 2000). Although variables differ between our analysis and these authors', the order of magnitude of Need and HI contributions remains the same.

\subsubsection{Comparison of results obtained with the standard and the new methodology}

The Need index found with our method differs largely from what is found with the standard methodology, whether in VanDoorslaer and Masseria (2004) or in our own calculations. For GP visits, the standard methodology generally finds on French data that the Need index amounts to almost $80 \%$ of $C$, while in our method Need represents roughly $20 \%$ of $C$.

In our results, the part of inequality due to differences in need is much smaller, and the part due to non-need variables (the unjustifiable part) is much bigger than what can be found with the standard methodology. The "unjustifiable" part of health care use inequality could thus be much larger than what the standard methodology indicates.

\subsubsection{Interpretation of our results: the importance of the heterogeneity of behaviors in the observed inequality}

We notice that $C<0$ for GP visits, and $C>0$ for specialist visits and the total number of visits. GP visits are thus concentrated among the poor, while specialist visits are concentrated among the rich. The Need index is negative for the three variables: needs are thus concentrated in the lowest part of the income distribution. The $H I$ index, that represents the degree of horizontal inequity, is negative for the number of GP visits and positive for the number of specialist visits and the total number of visits. This means that for GP visits, the poor receive more in proportion than the rich, 
for a given need. Conversely, for specialists visits, the rich receive more in proportion than the poor, for a given need.

The $H I$ index reflects both the distribution of variables and the heterogeneity of behaviors. The goal of our method is precisely to put emphasis on the behavioral component. We observe that inequality due to the heterogeneity of behaviors is far from negligible: for all type of care, it amounts to nearly half of the $H I$ index. Having estimated the model on each one of the income quintiles, we have shown that half of the horizontal inequity index is due to the heterogeneity of behaviors with respect to income. This observation cannot be identified in studies using the standard methodology.

We find that the effect of behaviors is globally pro-poor in the case of GP visits and pro-rich in the case of specialists visits. Each one of the three components of $H I$ have the same sign for each type of care. The heterogeneity of practices linked to participation is clearly pro-rich: for a given morbidity and given other characteristics, the poor resort less to physician care (GP or specialists) than the rich. However, the heterogeneity of behaviors linked to conditional consumption is propoor. Once the decision to consume is made, the poor receive more in proportion than the rich, for an equivalent morbidity.

For the three types of health care use we study (GP, specialists and total number of visits), the heterogeneity of practices linked to participation appears to be pro-rich. Participation behaviors for a given morbidity have a strong link to demand behaviors. This results shows the presence of a heterogeneity of access linked to the rank in the income distribution. This heterogeneity could signify the poor's lower preference for health. Indeed, the coefficients capturing practices for a given morbidity are estimated with a model where income is present, as well as many other individual characteristics. The influence of income level is thus taken into account in the explanatory model of health care use.

The heterogeneity of practices linked to conditional consumption appear to be pro-poor, for all types of health care use. Conditional consumption can be linked to the supply side. The suppliers' pro-poor behavior could compensate the pro-rich behaviors of individuals. The health care system would support equality of treatment. However, the poor's reduced participation in the health care system could induce a late recognition of their illness, and thus a degradation of health. This late participation could lead to more frequent and costly health care use once participation has been decided, and to less favorable outcomes overall.

The total number of physician visits is globally inequitable, and in favor of the rich $(H I=$ 0,0317). Half of this inequity arises from the heterogeneity of behaviors $(0,0182)$. This pro-rich 
inequity of behaviors is mainly due to the pro-rich heterogeneity of practices linked to participation that correspond to the demand side. The interpretation of results can be questioned: they can be interpreted as the poor's lower preference for health, or as an indication of a problem in access to care. In the case of a lower preference for health, should these preferences be followed?

As a matter of fact, the results we outline here can be strongly linked to the social epidemiology literature. The link between inequality in health and inequality in income is well recognised: according to Wilkinson (1992), the absolute level of income is less important that the relatve position of individuals in the society. From an economic point of view, this position can in some way be assessed by the rank of the individual in the income distribution. Our results showed heterogenous behaviors with respect to the individual rank in the income distribution in a model where income level is controlled. Following the social epidemiology literature, it indicates that when explaining individual behaviors, the relative place in the society is as important as the observed absolute income level. Within a public policy perspective, these results might indicate that in order to reduce inequity in health care use, one should reduce income inequality but also influence behaviors of underconsumption that can be observed in the lower part of the income distribution. Solely reducing income inequality will indeed reduce health care use inequity, but if behaviors are linked to the relative position in the society (which in our study is represented by the rank in the income distribution), an important source of inequity will remain. This source of inequity, the heterogeneity of behaviors with respect to social rank, cannot be identified in the standard decompositions, and its effect is unjustly attributed to inequality of income (as in expression (3)). Using the standard decomposition for policy purposes might be misleading, in the sense that it leads to neglecting an important source of inequity.

\section{Conclusion}

The method we propose for the analysis of inequality provides a synthetic analysis of the various factors that lead to the observed inequality in health care use. The micro-simulation approach avoids the computation and calculation of indices whose interpretation might be questionable, as in the standard decomposition. Our estimation by income quantile improves upon standard decompositions by providing the identification of heterogeneity of behaviors linked to the rank in the income distribution. While the standard way of testing for health care use inequity is usually done by comparing behaviors relative to various income quantiles (see Wagstaff and VanDoorslaer (2000)), this feature had remained absent from the standard decompositions of health care use. The decomposition we propose here provides a link between the Oaxaca (1973) style counterfactual analyses and the inequity decomposition methods. 
The application we proposed enabled us to analyze the sources of inequity in physician visits in France in 1998. Our results are consistent with the previous literature in that for a given morbidity, GP use is concentrated among the poor, and specialist use is concentrated among the rich.

We adhered to the standard approach in the literature that distinguishes justifiable and unjustifiable inequality. The index of horizontal inequity that we built $(H I)$ was decomposed into behaviors and individual characteristics; we showed that the heterogeneity of behaviors represents nearly half of the $H I$ index. Taking into account the heterogeneity of behaviors appears to be fundamental when trying to explain the sources of inequity in health care use. This heterogeneity of behaviors can be linked to the heterogeneity of individual preferences. The link with the social epidemiology literature improves upon the standard decomposition approaches, and further research should examine the heterogeneity of health care behaviors and its impact on inequity.

\section{References}

Dormont, B., M. Grignon, and H. Huber (2006): "Health expenditure growth: reassessing the threat of aging," Health Economics, 15, 947-963.

Grogger, J., and R. Carson (1991): "Models for truncated counts," Journal of Applied Econometrics, 6(3), 225-238.

OAXACA, R. (1973): "Male-Female Wage Differentials in Urban Labor Markets," International Economic Review, 14, 693-709.

VanDoorslaer, E., X. Koolman, and A. Jones (2004): "Explaining income-related inequalities in doctor utilisation in Europe," Health Economics, 13, 629-647.

VanDoorslaer, E., and C. Masseria (2004): "Income-related inequality in the use of medical care in 21 OECD countries," Equipe Française: A. Couffinhal, S. Tubeuf, P. Dourgnon , Irdes.

VanDoorslaer, E., A. Wagstaff, H. V. D. Burg, T. Christiansen, D. D. Graeve, I. Duchesne, U.-G. Gerdtham, M. Gerfin, J. Geurts, L. Gross, U. Hakkinen, J. John, J. Klavus, R. Leu, B. Nolan, O. O’Donnell, C. Propper, F. Puffer, M. Schellhorn, G. Sundberg, and O. Winkelhake (2000): "Equity in the delivery of health care in Europe and the US," Journal of Health Economics, 19, 553-583.

Wagstaff, A., and E. VanDoorslaer (2000): Handbook of Health Economicsvol. 1, chap. Equity in health care finance and delivery, pp. 1803-1862. Elsevier.

Wagstaff, A., E. VanDoorslaer, and N. Watanabe (2003): "On decomposing the causes of health sector inequalities with an application to malnutrition inequalities in Vietnam," Journal of Econometrics, 112, 207-223. 
Wilkinson, R. (1992): Unhealthy societies: the afflictions of inequality. London Routledge.

WorLdBAnK (2005): "Measuring inequity in health service delivery," http://siteresources . worldbank. org. 